\title{
Viscogonioplasty in narrow angle glaucoma: a randomized controlled trial
}

\section{Deepali Varma' \\ Wendy Adams' \\ Catey Bunce ${ }^{2}$ \\ Peter Phelan' \\ Scott Fraser}

'Sunderland Eye Infirmary, Queen Alexandra Road, Sunderland, Tyne and Wear, UK; ${ }^{2}$ Moorfields Eye Hospital, City Road, London, UK
Correspondence: Scott G Fraser Sunderland Eye Infirmary, Queen Alexandra Road, Sunderland, Tyne and Wear SR 2 9HP, UK Tel +44 (0) I I I 5699853 Fax +44 (0) I9I 5699856 Email sfraser 100@gmail.com
This article was published in the following Dove Press journal:

Clinical Ophthalmology

7 December 2010

Number of times this article has been viewed

Purpose: To determine the effect of viscogonioplasty and cataract extraction on intraocular pressure in patients with narrow angle glaucoma.

Methods: This was a double-masked randomized controlled trial involving 50 eyes ( 25 cases and 25 controls) from 38 consecutive patients. All patients underwent phacoemulsification with or without viscogonioplasty. The main outcome measures were intraocular pressure post-treatment and number of glaucoma medications post-treatment.

Results: Cases had a greater reduction in intraocular pressure than controls, with a mean intraocular pressure (standard deviation) at 12 months of $13.7( \pm 2.89) \mathrm{mmHg}$ compared with $16.2( \pm 3.55) \mathrm{mmHg}$ in controls $(P=0.009)$. Cases had a greater reduction in mean number of antiglaucoma medications than controls at 12 -month review, with 13 of 25 eyes $(52 \%)$ of cases controlled without any antiglaucoma therapy versus 9 of $25(36 \%)$ of the controls $(P=0.005)$.

Conclusions: Viscogonioplasty combined with cataract extraction has a significantly greater effect than cataract extraction alone on lowering intraocular pressure in patients with poorly controlled narrow angle glaucoma and should therefore be considered as a treatment option for patients with this condition.

Keywords: narrow angle, cataract extraction, viscogonioplasty, glaucoma

\section{Introduction}

Management of patients with poorly controlled narrow angle glaucoma due to extensive peripheral anterior synechiae (PAS) is difficult. Several glaucoma procedures have been advocated to open the angle in patients with extensive PAS, eg, surgical goniosynechiolysis ${ }^{1,2}$ and $\mathrm{Nd}$ :YAG laser-assisted goniosynechiolysis. ${ }^{3}$ These procedures have often been associated with significant side effects, including hemorrhage and damage to the angle. ${ }^{1-3}$ Viscogonioplasty, which is a technique for breaking PAS in acute angle closure glaucoma and chronic narrow angle glaucoma using a heavy viscoelastic, has previously been described by the authors. ${ }^{4,5}$

Phacoemulsification and implantation of a foldable intraocular lens in glaucomatous eyes can, in itself, decrease the intraocular pressure and the number of antiglaucoma medications required. ${ }^{6}$ However, the effect of phacoemulsification and intraocular lens implantation in patients with poorly controlled narrow angle glaucoma and PAS has not been extensively studied. One study did demonstrate a potential benefit from surgical goniosynechiolysis performed in conjunction with phacoemulsification, ${ }^{2}$ but this was a nonrandomized clinical trial. 
A pilot study using viscogonioplasty in combination with cataract extraction in patients with poorly controlled narrow angle glaucoma showed promising results, with a reduction in intraocular pressure, but the exact contribution of cataract removal in lowering intraocular pressure when combined with viscogonioplasty was unclear. ${ }^{4,5} \mathrm{~A}$ randomized controlled trial was therefore conducted to confirm the effect, if any, of viscogonioplasty combined with cataract extraction compared with cataract extraction alone on lowering intraocular pressure in patients with poorly controlled narrow angle glaucoma. To the best of our knowledge, no other studies have been performed examining this effect.

\section{Subjects and methods}

The study protocol followed the tenets of the Declaration of Helsinki and was approved by the local Sunderland research ethics committee. Informed written consent was obtained from every study patient after the nature and scope of the study protocol were explained. Each study patient was provided with a patient information sheet and helpline contact details.

\section{Patients}

Patients with chronic narrow angle glaucoma who were scheduled for cataract extraction between April 2006 and May 2007 were prospectively enrolled. All patients had an intraocular pressure that was judged to be too high, despite a patent iridotomy and use of antiglaucomatous medications.

Thirty-eight patients (50 eyes) who met the inclusion criteria were recruited for the study (Table 1). Patients were randomized using a random number generator to receive phacoemulsification with viscogonioplasty (25 cases) or phacoemulsification alone ( 25 controls). Patients were aged

Table I Inclusion and exclusion criteria

\begin{tabular}{|c|c|}
\hline Inclusion criteria & Exclusion criteria \\
\hline $\begin{array}{l}\text { - Occludable angle confirmed by } \\
\text { gonioscopy (defined as the inability } \\
\text { to see less than } 90^{\circ} \text { of the posterior } \\
\text { trabecular meshwork) } \\
\text { - Evidence of uncontrolled intraocular } \\
\text { pressure (raised intraocular pressure, } \\
\text { progressive glaucomatous optic } \\
\text { neuropathy, and/or visual field } \\
\text { progression) } \\
\text { Previous patent laser peripheral } \\
\text { iridotomy to eliminate pupillary block }\end{array}$ & $\begin{array}{l}\text { - Plateau iris syndrome } \\
\text { - Previous glaucoma surgery } \\
\text { (argon laser trabeculoplasty/ } \\
\text { trabeculectomy) } \\
\text { - Retinal disease } \\
\text { - History of ocular injury } \\
\text { - Other glaucomas }\end{array}$ \\
\hline
\end{tabular}

between 59 and 92 years, with a mean age of 72.40 years in the cases and 72.96 years in the controls (Table 2).

All patients underwent a complete ophthalmic examination by the same consultant ophthalmologist with an interest in glaucoma, including refraction, visual acuity testing, slit lamp biomicroscopy, applanation tonometry, fundus examination, and gonioscopy using a Zeiss four-mirror contact lens. Each patient's antiglaucomatous treatment was quantified using a scoring system devised by Kubota et $\mathrm{a}^{8}$ where 1 point is assigned for each antiglaucomatous topical treatment and 2 points for oral acetazolamide.

Patients were then randomized by a third party using a random number generator to receive either cataract extraction alone or cataract extraction with viscogonioplasty.

\section{Surgical intervention}

The same ophthalmic surgeon performed the surgical procedures in all study patients. All patients underwent routine phacoemulsification with intraocular lens implantation under topical anesthesia. Phacoemulsification was performed via a $3.2 \mathrm{~mm}$ temporal incision as a standard method, and a $5.5 \mathrm{~mm}$ foldable acrylic posterior chamber intraocular lens was implanted in the bag in all eyes. The surgeon did not know which group the patients were randomized to until after the phacoemulsification was completed, when he was instructed either to perform viscogonioplasty or to inject balanced salt solution into the angle.

\section{Technique of viscogonioplasty}

In patients receiving viscogonioplasty, a heavy viscoelastic was injected near the angle (without touching the trabecular meshwork) following intraocular lens implantation to break the PAS, taking care to avoid inducing ciliary body recession and inadvertent injury to the surrounding tissues. Care was also exercised while injecting the heavy viscoelastic in order to avoid inadvertent release of the cannula and damage to the drainage angle. No surgical instrument was used to physically break the PAS. Upon completion of

Table 2 Demographic characteristics of patients

\begin{tabular}{lll}
\hline Characteristics & Cases & Controls \\
\hline Eyes $(\mathrm{n})$ & 25 & 25 \\
Age $( \pm \mathrm{SD})$ in years & $72.4( \pm 8.9)$ & $72.96( \pm 7.8)$ \\
Gender & 16 male & 8 male \\
& 9 female & 17 female \\
Left/right & $11 / 14$ & $15 / 10$ \\
\hline
\end{tabular}

Abbreviation: SD, standard deviation. 
viscogonioplasty, viscoelastic was aspirated using automated irrigation/aspiration.

In order to duplicate every step in the cases and controls, balanced salt solution was injected into the angle, instead of the heavy viscoelastic, in the controls.

\section{Postoperative management and examination}

All patients received oral acetazolamide $250 \mathrm{mg}$ four times a day for 24 hours, as well as topical 1\% prednisolone acetate four times a day and topical ketorolac eye drops four times a day to the operated eye. Antiglaucoma medications were discontinued postoperatively and restarted according to intraocular pressure measurements following surgery.

One investigator examined all patients postoperatively on the first day, and at months 3, 6, and 12 postoperatively. The investigator was masked to the status (ie, cases or controls) of the study patients. At each postoperative examination, the intraocular pressure, number of antiglaucomatous medications, and gonioscopic angle appearance were recorded.

\section{Statistical analysis}

Analysis of parameters was conducted using analysis of variance $t$-tests, the Fisher exact test, or the Wilcoxon signed rank test as appropriate, depending on the variable analyzed.

\section{Results}

Baseline characteristics of both patient groups showed no evidence of difference in age, angle characteristics, antiglaucoma medication, and baseline intraocular pressure using analysis of variance (Table 2). No patients in the study had any complications associated with either cataract removal or viscogonioplasty.

\section{Intraocular pressure}

Table 3 summarizes the intraocular pressure results. From two weeks postoperatively, the intraocular pressure in the viscogonioplasty group was lower, despite the fact that the average intraocular pressure was higher preoperatively in this group. This difference increased up to 12 months, ie, the endpoint of the study. Using an analysis of covariance, adjusting from baseline intraocular pressure, there is evidence of a strong treatment effect at 12 months. Mean intraocular pressure (standard deviation) at 12 months postoperatively in cases was $13.7( \pm 2.89) \mathrm{mmHg}$ compared with $16.2( \pm 3.55) \mathrm{mmHg}$ in controls $(P=0.009)$.

\section{Glaucoma medications}

Thirteen of 25 eyes (52\%) cases were controlled without any antiglaucoma therapy versus 9 of $25(36 \%)$ in the controls. At 12-month review, there was a greater reduction in the mean number of antiglaucoma medications in cases than in controls $(P=0.005)$.

\section{Gonioscopy}

Gonioscopic measurements taken throughout the study indicated an improvement in angle width in cases compared with controls $(P<0.001)$. At the time of this study, anterior segment optical coherence tomography was not available, so the analysis was subjective.

\section{Discussion}

Eyes with narrow angle glaucoma can pose a considerable challenge. Many studies show that cataract extraction can normalize intraocular pressure in eyes with compromised outflow. This may be due to increased anterior chamber depth and consequent widening of the anterior chamber angle, but a proportion still have persistently high intraocular pressure following phacoemulsification, which either

Table 3 Preoperative and postoperative intraocular pressure after phacoemulsification/intraocular lens implantation and viscogonioplasty in cases and controls

\begin{tabular}{llll}
\hline & Mean IOP $( \pm$ SD $)(\mathbf{m m H g})$ & Controls \\
\cline { 2 - 3 } & Cases & $29.68( \pm 8.73)$ \\
\hline Highest recorded preoperative IOP on treatment & $30.12( \pm 7.03)$ & 20 \\
Immediate preoperative treated IOP & 23.8 & 15.88 \\
Two weeks postoperatively & 14.92 & 15.04 \\
Three months & 14.08 & 15.08 \\
Six months & 14.32 & $16.2( \pm 3.55)$ & 0.31 \\
I2 months & $13.7( \pm 2.89)$ &
\end{tabular}

Abbreviations: IOP, intraocular pressure; SD, standard deviation. 
requires long-term topical treatment or further surgical intervention. In the presence of uncontrolled glaucoma and coexisting cataract, the surgeon has three possible options: ie, performing cataract surgery alone, performing glaucoma surgery followed by later cataract surgery, or performing combined cataract and glaucoma surgery.

Glaucoma surgery for such cases has been substantially enhanced by the adjunctive use of antimetabolites in recent years, but vision-threatening complications, including hypotony, maculopathy, flat anterior chamber, and bleb infection, are not uncommon, and therefore suitable alternatives would be desirable.

PAS are acquired adhesions between the corneoscleral layer and the peripheral iris. They are seen as the hallmark of primary angle closure, and the extent of PAS correlates with the likelihood of glaucomatous optic neuropathy in this condition.

Several glaucoma procedures have been advocated to open the angle in patients with extensive PAS, but these procedures have been associated with significant side effects. Goniosynechiolysis is one such surgical procedure where PAS are broken in order to restore trabecular filtration. Conventionally, this has been performed using surgical instruments or by $\mathrm{Nd}: \mathrm{YAG}_{\text {laser. }}{ }^{1-3}$ However, complications reported with this procedure include mild to severe hemorrhage from the iris or trabecular meshwork, fibrin exudation, choroidal hemorrhage, choroidal detachment, shallow anterior chamber, and transient elevation of intraocular pressure in the immediate postoperative period.

We have previously shown, in eyes with acute angle closure glaucoma, the efficacy of performing viscogonioplasty following cataract extraction to produce further intraocular pressure reduction. ${ }^{4}$ In a further study in narrow angle glaucoma patients, phacoemulsification combined with viscogonioplasty appeared to not only lower intraocular pressure in the immediate postoperative period but also maintain a sustained effect over a significant period of time. ${ }^{5}$ However, due to lack of controls, it was difficult to conclude whether it was cataract surgery itself or the additional procedure of viscogonioplasty that offered the significant lowering of intraocular pressure.

This current randomized controlled trial has allowed us to examine our original findings and has made it clear that although some lowering of intraocular pressure is seen following cataract surgery alone, in eyes with narrow angles an additional lowering of intraocular pressure is achieved with simultaneous viscogonioplasty. In addition, we have demonstrated a reduction in the number of drops required to maintain lower intraocular pressure.

Gonioscopic appearance showed an improvement in appearance of the drainage angle in both groups but noticeably more in the viscogonioplasty group. We have shown an overall improvement in appearance on gonioscopic views, which is encouraging, but this was unfortunately subjective and will require a repeat study using anterior segment optical coherence tomography.

Our study does have some limitations in that our follow-up data published here were measured over a 12-month period. We are continuing to monitor our patients' intraocular pressure for a longer period to demonstrate stability with and without treatment. We are also, in the longer term, looking at any differences in visual field loss between the two groups.

One other limitation is that the technique used for viscogonioplasty is not in widespread use, and we are aware that if the findings from our paper are to be used in general ophthalmic practice then there will be a learning curve in the provision of this treatment.

\section{Conclusion}

This study demonstrated a pronounced and prolonged intraocular pressure reduction following cataract extraction and viscogonioplasty in eyes with chronic narrow angle glaucoma compared with cataract extraction alone. We suggest that because of viscogonioplasty's ease, safety, and potential benefit, eyes with narrow angles undergoing cataract extraction should have viscogonioplasty following intraocular lens implantation.

\section{Disclosure}

The authors report no conflicts of interest in this work.

\section{References}

1. Mirshahi A, Scharioth GB. Nonpenetrating glaucoma surgery with goniosynechiolysis ab interno: A surgical technique. Eur J Ophthalmol. 2009; 19:147-502.

2. Senn P, Kopp B. Nd:YAG laser synechiolysis in glaucoma due to iridocorneal angle synechiae. Klin Monatsbl Augenheilkd. 1990;196: 210-213. German.

3. Teekhasaenee C, Ritch R. Combined phacoemulsification and goniosynechiolysis for uncontrolled chronic angle closure glaucoma, after acute angle closure glaucoma. Ophthalmology. 1999;106:669-674.

4. Varma D, Adams WE, Phelan PS, Fraser SG. Viscogonioplasty in patients with chronic narrow angle glaucoma. Br J Ophthalmol. 2006; 90:648-649.

5. Varma D, Baylis O, Wride N, Phelan PS, Fraser SG. Viscogonioplasty: An effective procedure for lowering intraocular pressure in primary angle closure glaucoma. Eye. 2007;2:472-475. 
6. Hayashi K, Hayashi H, Nakao F, Hayashi F. Effect of cataract surgery on intraocular pressure control in glaucoma patients. J Cataract Refract Surg. 2001;27:1779-1786.

7. Foster PJ, Buhrmann RR, Quigley HA, et al. The definition and classification of glaucoma in prevalence surveys. Br J Ophthalmol. 2002; $86: 238-242$
8. Kubota T, Touguri I, Onizuka N, Matsuura T. Phacoemulsification and intraocular lens implantation combined with trabeculotomy for open-angle glaucoma and coexisting cataract. Ophthalmologica. 2003; 217:204-207.

\section{Publish your work in this journal}

Clinical Ophthalmology is an international, peer-reviewed journal covering all subspecialties within ophthalmology. Key topics include: Optometry; Visual science; Pharmacology and drug therapy in eye diseases; Basic Sciences; Primary and Secondary eye care; Patien Safety and Quality of Care Improvements. This journal is indexed on

Submit your manuscript here: http://www.dovepress.com/clinical-ophthalmology-journal

\section{Dovepress}

PubMed Central and CAS, and is the official journal of The Society of Clinical Ophthalmology (SCO). The manuscript management system is completely online and includes a very quick and fair peer-review system, which is all easy to use. Visit http://www.dovepress.com/ testimonials.php to read real quotes from published authors. 